\title{
Segmentation of RBC in Blood Smear Image using Discrete Shearlet Transform
}

\author{
Krishna Prasad Palli \\ Asst Professor \\ Vasireddy Venkatadri Institute \\ of Technology, Nambur
}

\author{
Sreenivasa Reddy Edara \\ Prof \& Dean \\ College of Engineering \\ Acharya Nagarjuna University
}

\author{
ChandraSekharaiah K. \\ Professor \\ JNTUH \\ Hyderabad
}

\begin{abstract}
Blood cell analysis is a critical step in the process of disease analysis. Blood analysis process, usually done manually, has been automated to overcome the cumbersome task of RBC identification, segmentation, and classification. The digital image processing techniques developed in the past few years has made the automation possible. Among all the levels of medical imaging of the blood cells, the segmentation of the blood cell is the most vital task. This paper attempts to develop a new technique to segment an RBC from blood smear images. The proposed method is implemented by extracting a color image from the light microscopic smear image. A green channel is extracted from the color image. Further, image filtering is used to remove noise from the captured image. Finally Red Blood Cell segmentation is implemented using discrete shearlet transform. The proposed method is tested on blood cell images.
\end{abstract}

\section{General Terms}

Here terms used are Median filtering, Color Image processing, discrete Shearlet transformations and other morphological operations in Medical Image processing for Image Segmentation et. al.

\section{Keywords}

Discrete Shearlet transform, median filtering, Red Blood Cell, Image Segmentation and Smear Image are the keywords used in the paper.

\section{INTRODUCTION}

Advancement in technology has revolutionized the field of medicine. One of the most important changes that took place is in the area of diagnosis. Technology has been helping the doctors to diagnose diseases quickly and at an early stage. Medical imaging has given the practitioners the advantage of saving time and diagnosing the disease accurately. Continuous research taking place in the field of medical image processing has been steadily giving some of the best solutions for the diagnosis challenges.

Advancements in medical imaging resulted in the development of tools that can assess pathological features effectively thus enabling the doctors to diagnose a disease in its early stage and provide suitable therapy. Analysis of blood cells is one of the prime diagnosis methods which if aided by novel medical imaging processes will yield effective results. The Blood in human tissues consists of WBC, RBC and Platelets. The RBC is recognized by the presence of Hemoglobin. The level of hemoglobin in the human blood will affect the health by causing various diseases. As the Blood cells convey more information, the process of Cell identification and segmentation plays a vital role in the diagnosis of a disease.
Blood consists of lot various components. The hemoglobin is responsible for the Red color in the blood. Obtaining information about the blood cell images in human tissue is essential in determining, the existence or the stage of the disease. These images convey a wealth of information that aids the experts. Obtaining such valuable information proves crucial in the diagnosis of many diseases. The manual blood cell analysis methods in place provide information that sometimes cannot identify the existence of a disease or the stage of the diseases like cancer.

The available digital image processing techniques assist in the process of accurate diagnosis of diseases. But, there are still some challenges in extracting data from Red blood cells. The variations in the position, shape, size and edge of the red blood cells makes the process of extraction difficult. Unevenness in illumination and variance in contrast between cell boundaries and the background during the capturing process further makes the process of extraction tough.

Hematologists use the image processing techniques developed in the last few years, to automatically process blood slides for the first level of screening of the blood samples to detect diseases. These techniques can become more effective if the segmentation procedures followed are updated. This paper proposes a shearlet transform technique that consists of multiple stages of segmentation, which can improve the feature extraction.

The figure 1.1 consists of $\mathrm{WBC}, \mathrm{RBC}$ and platelets. More clearly the figure 1.2 indicates RBC and WBC as well. It is really a tedious job to identify and make a count and to analyze the peripheral smear images [1].

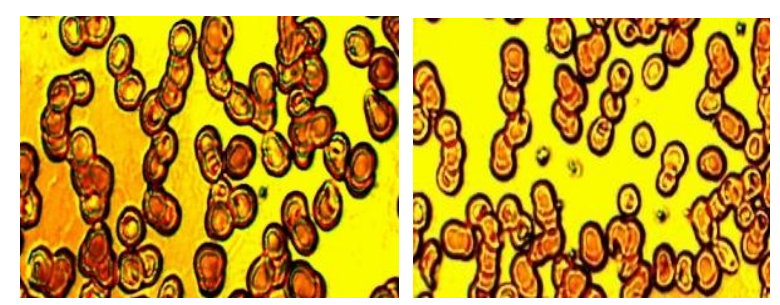

Fig 1 Blood Smear Images $(1,2)$

\section{LITERATURE SURVEY}

The area of medical image processing witnessed considerable amount research in the past few years. It has been studied that the three major stages of blood cell image analysis in medical imaging $v i z$, image acquisition, noise removal and segmentation are strengthened by the application of various new algorithms developed. The proposed discrete shearlet transform method is an effective extension to the existing methods. In this work the techniques followed in the Noise removal and Image Segmentation stages are studied and it is 
understood that more effective methods can be proposed to make blood cell analysis a comprehensive method.

\subsection{Noise Removal}

Images are usually degraded by various noises in the signal transmission, coding and decoding processing. The results of image processing such as image segmentation, feature extraction and image recognition will, to a great extent, depend on the noise removal results. So de-noising an image is of great importance in image processing [13]. Currently, the advances in mathematics and its' applications has been critical to the research and development of digital image processing. Normally the median filter is a choice to remove noise in the images. In this approach, each pixel in the image and its nearby neighboring pixels to decide whether or not it represents its surrounding pixels. Instead of simply replacing the pixel value with the mean of neighboring pixel values, it replaces it with the median of those values. The median is evaluated by first sorting all the pixel values from the surrounding neighborhood into numerical order and then replacing the pixel being considered with the middle pixel value. This may avoid false operation on non noise pixels.

\subsection{Image Segmentation}

Image segmentation is the most important step and a key technology in image processing, and it will directly affect subsequent processing. With mathematical theories, image segmentation has achieved great progress and a lot of novel segmenting algorithms have been proposed. Several works have been conducted in the area of general segmentation methods. Among the common segmentation methods are edge and border detection, region growing, filtering, mathematical morphology, and watershed clustering, J M Sharif et al. [2], Usha Rani et al. [6] did segmentation by using Watershed algorithm. Kumar et al. [3] used teager energy operator for segmentation, C Rongtai et al. [4] introduced Active Appearance Model. Venkatalakshmi B et al. [5] worked on Hough Transforms.

\section{PROPOSED SYSTEM}

An effective method is required to segment RBC from the smear image. The proposed system emphasizes on discrete shearlet Transform. Initially an RGB blood smear image is taken and it is converted into CIE L*a*b. Further image enhancement technique is applied to go for further step, segmentation. The steps given in the figure below:

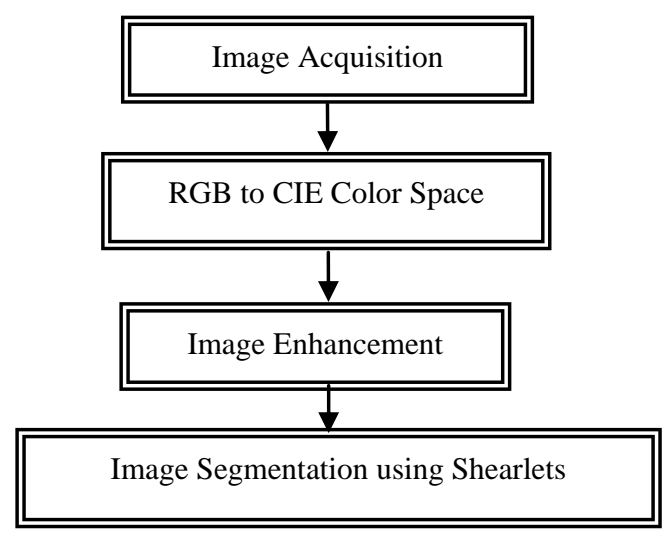

Fig 2. the steps involved in RBC Segmentation

\subsection{CIE L*a*b* Color Space}

Color space defined by the CIE, based on one channel for Luminance (lightness) (L) and two color channels (a and b). The CIE L*a*b Space can be used if color perception is given importance in the image analysis. The $\mathrm{L}^{*} \mathrm{a} * \mathrm{~b} *$ space consists of a luminosity ' $\mathrm{L}$ '' or brightness layer, chromaticity layer ' $\mathrm{a}^{*}$ indicates where color falls along the red-green axis, and chromaticity layer ' $b$ *' indicates the color falls along the blueyellow axis. The RGB color space can be transformed to the CIE L*a*b* color space as follows:

$$
\begin{gathered}
{\left[\begin{array}{l}
x \\
y \\
Z
\end{array}\right]=\frac{100}{250} \times\left[\begin{array}{lll}
0.412530 & 0.357580 & 0.180423 \\
0.212671 & 0.715160 & 0.072169 \\
0.019334 & 0.119193 & 0.950227
\end{array}\right]} \\
\times\left[\begin{array}{l}
R \\
G \\
B
\end{array}\right](1) \\
L^{*}=116 f\left(\frac{Y}{Y_{0}}\right)-16 \\
a^{*}=500\left[f\left(\frac{X}{X_{0}}\right)-f\left(\frac{Y}{Y_{0}}\right)\right] \\
b^{*}=200\left[f\left(\frac{Y}{Y_{0}}\right)-f\left(\frac{Z}{Z_{0}}\right)\right]
\end{gathered}
$$

\subsection{Mean Shift Filter}

Mean shift filter is a powerful tool and it is used for noise removal. Mean Shift Filter Let $X=\{x\}_{i=1}{ }^{n}$ be a set of $n$ data points in d-dimensional space, Rd. The multivariate kernel density estimator with Gaussian kernel and a symmetric positive definite dxd bandwidth matrix $\mathrm{H}$, computed at the point $\mathrm{x}$ is given by

$\hat{f}(x)=\frac{1}{n|2 \pi H|^{\frac{1}{2}}} \sum_{i=1}^{n} \exp \left(-\frac{1}{2} d^{2}\left(x, x_{i} H\right)\right)$

Where

$d^{2}\left(x, x_{i}, H\right) \equiv\left(x-x_{i}\right)^{T} H^{-1}\left(x-x_{i}\right)$

is the Mahalanobis distance from $\mathrm{x}$ to $\mathrm{xi}$. By computing the gradient of $\mathrm{f}(\mathrm{x})$

$\nabla \hat{f}(x)=\frac{H^{-1}}{n|2 \pi H|^{\frac{1}{2}}} \sum_{i=1}^{n}\left(x_{i}-x\right) \times \exp \left(-\frac{1}{2} \mathrm{~d}^{2}(\mathrm{x}, \mathrm{xi}, \mathrm{H})\right)$

After some algebra we have

$$
\begin{aligned}
& \mathrm{m}(\mathrm{x})=\mathrm{H} \frac{\nabla \hat{f}(x)}{\hat{f}(x)} \text { (6) Where } \\
& \mathrm{m}(\mathrm{x})=\frac{\sum_{i=\mathbf{1}}^{n}\left(\mathbf{x}_{\mathbf{i}}\right) \exp \left(-\frac{1}{2} \mathbf{d} 2(\mathbf{x}, \mathbf{x i}, \mathbf{H})\right)}{\sum_{i=\mathbf{1}}^{n} \exp \left(-\frac{1}{2} \mathbf{d} 2(\mathbf{x}, \mathbf{x i}, \mathbf{H})\right)}-\boldsymbol{x}
\end{aligned}
$$

is the mean shift vector [7].

Assume now that the data points xi are extracted from an input image. Then the vector components of xi contain both the spatial lattice information $\mathrm{xsi}=(\mathrm{xi} ; \mathrm{yi}) \mathrm{T}$ and range information $\mathrm{xci}=(\mathrm{ci} 1 ; \mathrm{ci} 2$; $\mathrm{ci} 3) \mathrm{T}$ where $\mathrm{ci} 1$; $\mathrm{ci} 2$ and ci3 are three color components at position $(x i ; y i)$. Then $x i=(x T$ si; $\mathrm{xTri}) \mathrm{T}$ is a data point in joint partial-range domain. We assume that the bandwidth matrix $\mathrm{H}$ is diagonal having the diagonal terms equal to _2S for the spatial part and _2R for the range part. Then the Mahalanobis distance in Equation (4) can be rewritten as: 
$\mathrm{D}^{2}\left(\mathrm{x}, \mathrm{x}_{\mathrm{i}}, \mathrm{H}\right) \equiv \frac{\left\|x_{s}-x_{s i}\right\|^{2}}{2 \sigma_{S}^{2}}+\frac{\left\|x_{r}-x_{r i}\right\|^{2}}{2 \sigma_{R}^{2}}$

where

$$
\left\|x_{r}-x_{r i}\right\|^{2} \equiv\left(r-r_{i}\right)^{2}+\left(g-g_{i}\right)^{2}+\left(b-b_{i}\right)^{2}
$$

\subsection{Segmentation using Discrete Shearlet Transform}

Shearlet transforms is a general framework for analyzing and representing data with anisotropic information at multiple scales. As a result the properties like edges can be precisely detected and located in images [13]. The approach for shape used in this paper is based on a new multi-scale transform called the shearlet transform. It is a multidimensional version of the traditional wavelet transform, and is especially designed to address anisotropic and directional information at various scales. Indeed, the traditional wavelet approach, which is based on isotropic dilations, has a very limited capability to account for the geometry of multidimensional functions. In contrast, the analyzing functions associated with the shearlet transform are highly anisotropic, and, unlike traditional wavelets, are defined at various scales, locations and orientations. As a consequence, this transform provides an optimally efficient representation of images with edges [10]. The shearlet transform has similarities to the curvelet transform, Shearlets and curvelets, in fact, are the only two systems which were mathematically known to provide optimally sparse representations of images with edges and the implementations of the curvelet transform correspond to essentially the same tiling as that of the shearlet transform. Both systems are related to contourlets $[14,15]$ and steerable filters [16]. Contourlets, however, provide a purely discrete approach which presents difficulties in rigorously addressing the edge detection problem. We refer to $[9,10]$ for more details about the comparison of shearlets and other orientable multi-scale transforms.

In this paper, we combine the shearlet framework with several established ideas from the image processing literature to obtain improved and computationally efficient algorithms for edge analysis and detection. The present approach may be viewed as a truly multidimensional refinement of the approach of Mallat et al., where the isotropic wavelet transform is replaced by an anisotropic directional multi-scale transform. As a result, the well known shearlet transform acts like a multi-scale directional difference operator and provides a number of very useful features with improved accuracy in the detection of edge orientation. Using anisotropic dilations and multiple orientations, the shearlet transform precisely captures the geometry of edges. It is a multi-scale transform, based on the same affine mathematical structure of traditional wavelets and Computational efficiency. The discretization of the shearlet transform provides a stable and computationally efficient decomposition and reconstruction algorithm for images.

The examination will start by considering the behavior of the continuous shearlet transform of functions of the form, where $\mathrm{F}=\mathrm{X}_{\mathrm{c}}$ is the characteristic function of a planar region. This can be seen as a model of an image containing a step edge along the curve described by the boundary of C. Since the properties of the "horizontal" continuous shearlet transform are essentially identical to those of the "vertical" continuous shearlet transform, in the following, it will be sufficient to examine the horizontal transform only $[11,12]$.

\section{EXPERIMENTAL RESULTS}

To evaluate the technique initially blood smears are collected and stained. The Stained smear images are observed through a Microscope. The blood smear images are converted into digital images and experiments are done on a set of 50 images where sub images have been considered to testing. The images are collected from the Maata Labs. Here Motic Microscopic Images are taken into consideration. The images normally contain RBC, WBC and platelets.

Normally RBC count is higher than WBC. More over there is difference in shape of RBC and WBC. Platelets are irregular in shape and smaller in size compare to RBC and WBC. They are ignored in the staining process. To visualize and differentiate both $\mathrm{RBC}$ and $\mathrm{WBC}$, staining is done at early stage.

The figure 4.1 visualizes the actual blood cell and segmented images. In figure 4.1, two sets of images are shown. Figures $(1,2,3)$ represent one set of images and $(4,5,6)$ represent another set of images.

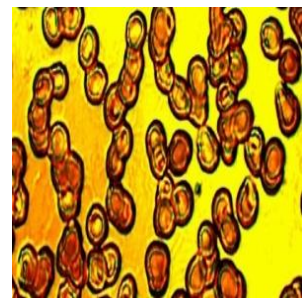

(1)

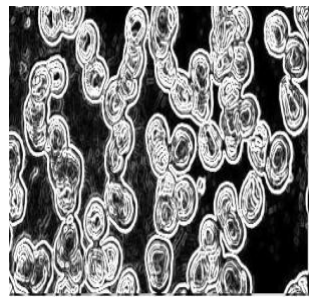

(2)

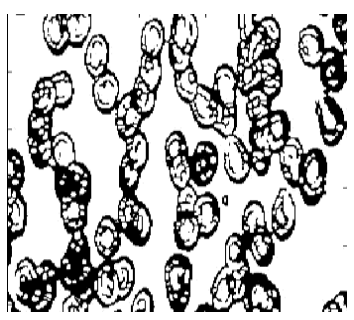

(3)

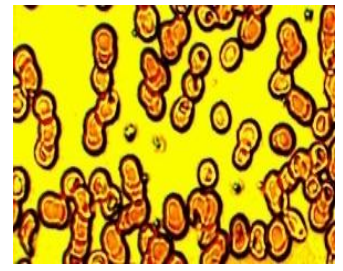

(4)

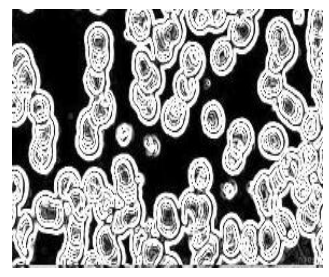

(5)

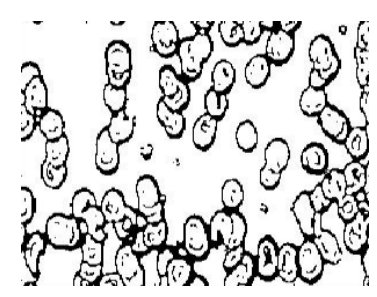

(6)

\section{Fig. 4.1 Original Blood Cell \& Segmented Image}

The Segmented Red Blood Cell images will be compared with the manual identification and segmentation. Here the ground truth is taken into consideration. The ground truth is evaluated 
with the help of expert in hematology. Usually it is a difficult process for evaluating the efficiency of any segmentation process. The comparison can be made using human evaluation and machine evaluation to assess the quality of the Image segmentation.

\section{CONCLUSION AND FUTURE SCOPE}

This paper has demonstrated a new discrete shearlet transform for segmenting Red blood cells and using integration of other concepts in Digital Image Processing. The proposed technique is able to yield more accuracy for segmentation. Further the images after segmentation may undergo feature extraction and classification as medical images require more attention.

\section{ACKNOWLEDGMENTS}

It is mandatory to thank the experts Bhavani and Sudhakar who have contributed towards development of the paper.

\section{REFERENCES}

[1] RitterNand Cooper J (2007) Segmentation and border identification of cells in images of peripheral blood smear slides. In Proceedings of the Thirtieth Australasian Conference on Computer Science 62:161-169

[2] J. M. Sarif, M. Miswan, M. Ngadi, M. S. H. Salam, and M. Mahadi bin Abdul Jamil, "Red blood cell segmentation using masking and watershed algorithm: A preliminary study in Biomedical Engineering (ICoBE), 2012 International Conference on, 2012, pp. 258-262.

[3] Kumar BR, Joseph DK and Sreenivasan TV (2002) Teager energy based blood cell segmentation. Digital Signal Processing. DSP 2002 14th International Conference on, vol. 2:619-622

[4] C. Rongtai, W. QingXiang, Z. Rui, F. Lijuan, and R. Chengmei, "Red blood cell segmentation using Active Appearance Model," in Signal Processing (ICSP), 2012 IEEE 11th International Conference on, 2012, pp. 16411644.

[5] Venkatalakshmi, B. and K. Thilagavathi. Automatic red blood cell counting using Hough transforms, 2013 IEEE Conference on Information \& Communication Technologies (ICT). 2013.
[6] Sumeet Chourasiya, G Usha Rani (2014) "Automatic Red Blood Cell Counting using Watershed Segmentation" IJCSIT.

[7] Razali Tomari, Wan Nurshazwani Wan Zakaria, Mouhammad Mahadi Abdul Jamil, Faridah Mohd Nor, Nik Farhan Nik Faud, "Computer Aided System for Red Blood Cell Classification in Blood Smear Image" in International Conference on Robot PRIDE, Elsevier, 2014.

[8] Prof Samir K Bandyopadhyay, "Method for Blood Cell segmentation", JCRCS, vol. 2, No. 4, 2011.

[9] G. Easley, D. Labate, and W-Q. Lim "Sparse Directional Image Representations using the Discrete Shearlet Transform", to appear in Appl. Comput. Harmon. Anal. 2008.

[10] K. Guo and D. Labate, "Optimally sparse multidimensional representation using shearlets", SIAM J. Math. Anal., Vol.9, pp. 298-318, 2007.

[11] K. Guo and D. Labate, Characterization and analysis of edges using the continuous shearlet transform, SIAM J. Imaging Sciences 2 (2009), 959-986.

[12] K. Guo, D. Labate and W. Lim, Edge analysis and identification using the continuous shearlet transform, Appl. Comput. Harmon. Anal. 27 (2009), 24-46.

[13] S. Yi, D. Labate, G. R. Easley, and H. Krim, Edge detection and processing using shearlets, Proc. IEEE Int Conference on Image Proc., San Diego, October 12-15, 2008.

[14] M. N. Do, and M. Vetterli, "The contourlet transform: an efficient directional multi-resolution image representation", IEEE Trans. Image Process., vol. 14, no. 12, pp. 2091-2106, Dec. 2005.

[15] D. D. Po and M. N. Do, "Directional multiscale modeling of images using the contourlet transform", IEEE Trans. Image Processing., vol. 15, no. 6, pp. 16101620 , June 2006

[16] W. Freeman and E. Adelson, "The design and use of steerable filters", IEEE Trans. Patt. Anal. and Machine Intell., Vol. 13, pp. 891-906, 1991. 